ritative or inflammatory symptoms does not cure the disease. In the presence of the pressure exerted by the swollen vitreous against the cilio-iridic recrion, the ciliary muscle readily tires (witness the easily produced eyestrain of the simple glaucomatous eye). This functional exhaustion disposes the ciliary region to active congestion, if the blood contains an undue proportion of toxins from foci of infection. This congestion may be slight or severe and hence the irritative or the socalled inflammatory types. Remozal of the foci of infection, if done in time, is followed by disappearance of the irritative or inflammatory stage, but the process known as simple glaucoma remains present as before. We have in the above the explanation of the socalled cures of acute glaucoma following extraction of a tooth, or opening and draining of a nasal air chamber, which from time to time have been reported in medical literature.

\title{
CLINICAL OBSERVATIONS ON INCREASED INTRAOCULAR TENSION.
}

\author{
Thomas Faith, M.D., \\ CHICAGO, IILLINOIS.
}

The differentiation between glaucoma and uveitis with increased intraocular tension is sometimes very difficult, altho text-books fail to point this out. This paper reports a series of cases in which the tension was reduced to normal and permanent cure secured by the use of mydriatics. Read before the Chicago Ophthalmological Society, February 20, 1922. See p. 379 .

It is not the intention of this paper to open a general discussion on the subject of glaucoma, but to review a study of the following cases which will illustrate the difficulty frequently encountered in differentiating some of the cases of uveal inflammation which come under observation from cases of primary glaucoma. If we read either a modern text-book on ophthalmology, or one published twenty years ago, the conclusion arrived at will be that the two conditions-iridocyclitis and glaucoma-have well marked differential points, which enable one to make a diagnosis at once. Yet this is a question which often grives not only the student but also the trained ophthalmologist the most concern in practice. Time and again this question presents itself, and we are all called upon to make a decision which is difficult as well as extremely important.

Classical cases are, of course, readily recognized, but the atypical cases are the difficult as well as the interesting ones. We are all aware that every eye which has increased intraocular tension is not affected with glaucoma, and we should keep this fact firmly fixed in our minds, since it often happens that increased hardness of the eyeball is such an outstanding feature in the case under observation that it scems to engage our entire attention, even to the exclusion of all other symptoms, and primary glaucoma is our first and only thought; and following on in this direction miotic treatment is at once resorted to, sometimes with disasterous results, whereas mydriatic treatment would have saved the day. The following cases are illustrative of this point and are submitted for that purpose.

Case 1. Many years ago a young ma11 22 years of age came under my observation, who had been suffering with severe pain in the left eye for a number of days. The jain was so severe when I first saw him that he was nauseated and had been vomiting most of the time during the previous days. I found his pupil partly dilated, the anterior chamber practically obliterated, eyeball hard, and the lens somewhat opaque. Ile had been struck on the eye by the ramrod of a toy pistol about twelve days previously. There was apparently no rupture of the anterior capsule of the lens and no lens matter could be seen in the anterior chamber.

I reasoned that the capsule had been ruptured posteriorly and that the lens swelling had pushed the iris forward 
and obliterated the sinus angle, and since his lens had not attained its complete growth at 22 years of age, I would attempt complete dilatation with atropin, and if that could be accomplished it would restore drainage from the posterior to the anterior chamber and probably open up the sinus angle.

I accordingly began with alternate instillations of cocain and atropin, and within four or frve hours his tension and pain were relieved and he ran the usual course of a case of traumatic cataract, except that there never was any lens matter in the anterior chamber at any time, absorption taking place posteriorly until there was nothing but a capsule remaining. This was my first experience of this kind and I was very much impressed with the fact that altho the swollen lens was not removed, the hypertension was relieved immediately and permanently by the use of atropin.

Cise 2. In the spring of 1898 a man of 35 years came to me with a well marked iridocyclitis. Ile had a history of lues and many corruborative symptoms of the discase. He was put on atropin and specific medication and made very satisfactory procress for about three weeks, pupil dilated readily and evenly and his pain was relieved. Then the eye suddenly bocame painful anl tender and the tension became $-f$. Ieeching was resorted to, but without relief of either pain or tension. Atropin was suspended and the pupil rapidly contracted; at the same time the anterior chamber became deeper than previously, the aqueous became cloudy and the tension and pain increased. Atropin was resumed with some improvement in the tension and was pushed vigorously; the pupil dilated rather surprisingly and there appeared to be no adhesions whatever, but numerous fine precipitates on the lens and posterior surface of the cornea. A paracentesis was done with almost magic relief, tension remaining down and recovery promptly taking place. Evidently a case of what we used to call serous iritis, the turbid aqueous being the probable cause of the hypertension.
Case 3. $\Lambda$ babe of $21 / 2$ years brought with congenital cataract in both eyes. Right eye needled and three wecks later left eye needled. Eight or ten weeks later right eye necdled again with rapid absorption of lens matter for a number of weeks, then pupil became difficult to keep dilated and later tension became elevated and the eye became red and painful. At this time the anterior chamber was quite shallow. tho there seemed to be nothing but capsule remaining. A capsular discission was made on the theory that the iris was adherent to the capsule and chamber communication shut off. No relief was afforded by the opening thru the capsule and two days later an iridectomy was done. $A$ very free gush of aqueous followed the $a b$ scission of the iris, and relief was prompt and permanent.

Here apparently the aqueous was secreted into a closed pocket formed by the iris anteriorly, the thickened lens capsule and zonule posteriorly and nearly or quite surrounding the pupil. After the iridectomy the pupil dilated readily with atropin and a good result was secured. The abore cases antedate the tonometer, thus tonometer records were not made.

Case 4. Mrs. M. S., agge 43. Came complaining of blurred vision in left cye for previous week, with occasional severe pain above the eye; had similar trouble sixteen or seventeen years ago. R.V. $=20 / 25$, L.V.V $=20 / 100$; I. eye congested, anterior scleral vessels much distended and pupil slightly dilated; reaction to light sluggish, anterior chamber deep, $T$ decidedly + , media clear and fundus normal. $R$. field normal, left field contracted $20^{\circ}$ in nasa! portion; with tonometer $T:=75$. Such high tension with pupillary reaction was so unusual that I secured another tonometer, thinking my instrument might be at fault, and on restoring found $\mathrm{T}=75$, the same as with 'my own Gradle tonometer. Arterial pulsation was not present, but could be produced by pressure on the globs. There was a small posterior syrach:a below, which the patient stated hid been present since the first attack of the trouble. 
On account of the deep anterior chamber I decided to carefully use a mydriatic and accordingly applied five instillations of $2 \%$ homatropin with two instillations of $4 \%$ cocain. At the end of ninety minutes the pupil was dilated widely, and $T$. was reduced to 60 , a drop of $15 \mathrm{~mm}$. A careful examination of the cornea, lens and media showed them to be entirely free from exudates. Scopolamin hyd. brom. grs. 1 to $\overline{5} 1$ was ordered every two hours. a leech was applied to the temple and a course of calomel and saline was griven. The next day $T==50$, less pain and blurring, and in four days tension was 24 , with vision $20 / 30$, and entire frecdom from pain. The tension remaincd at this point for one week when it decreased farther recording 10 $\mathrm{mm}$. with the same tonometer. Abont this time a few deposits appeared on the lens and in the vitreous, but none could be seen on the posterior surface of the cornea. This patient had a ++++ Wassermann and a number of dead teeth with apical infection. But she had practically recovered from her eye condition before any of the teeth were extracted. She had been given about eight or ten rubs of ung. hydrarg. up to this time. The eye remained well and she had her bad teeth removed as well as continuing her treatment for lues. When last seen by me, which was about nine weeks after the onset of her trouble, her vision was $20 / 25$, $\mathrm{T}=18$ to 20 and field of vision normal.

Case 5. Mr. R., age 24. Came with a history of loss of vision in his left eye dating back about two months. The eye showed evidence of inflammation and was quite painful at times; the anterior chamber was shallow, pupil was contracted, and there were $\therefore$ few deposits on the posterior surface of the cornea, at from five to seven o'cleck. A distinct circumcorneal injecticn was present and tension was $54 \mathrm{~mm}$.

This patient had in his possession a $2 \%$ sol. oi vilocarpin, and $5 \%$ sol. of dionin, whic's had been ordered by a local ophthaimologist. Vision was light and shadow's only, and fundus could not be seen on account of the contracted pupil and the great number of vitreous opacities which were present. A diarrnosis of uveitis was readily made and the use of a mydriatic was determined upon. Accordingly homatropin $2 \%$ was ordered for instillation every hour, the patient was leeched and put to bed.

I saw him again in the evening and found the pupil fairly well dilated and the tension somewhat reduced. Scopolamin was substituted for homatropin and the next day tension had dropped to 42 ; scopolamin was continued for another 24 hours and was then replaced by atropin, the tension having dropped to 30 . In the meantime a blood Wassermann showed negative, and a badly diseased pair of tonsils were found as the only apparent cause of the trouble. These were removed and a rapid recovery from the acute process followed, leaving only the results of inflammatory exudation, which slowly disappeared with the exception of the vitreous opacities, but with no return of the hypertension. When last seen vision $=20 / 65$ with -.75 sphere. This was about three months after his first visit to me. There were quite a few floaters remaining in the vitreous at this time. Two and a half years later this patient had vision of $20 / 25$ with $-1.25=-.75$ ax. 165. No vitreous opacities present.

From a study of these cases it will be seen that the anterior chamber may be either shallow or deep, depending on the pathology present. If the lens or lens capsule is involved, or if there is posterior or peripheral anterior synechia the chamber will appear shallow. This is particularly true if the disease has been present for some time before proper mydriatic treatment has been instituted, or if it has been difficult to obtain proper absorption of the drug; while the chamber is usually deep in the carlier stages of the disease, which condition is probably due to the freedom of communication between posterior and anterior chambers, which exists at this time, and an increase in the albuminous contents of the aqueous, which varies considerably in different cases. 
The pupil may be dilated to some extent, as in cases one and five, but is most of ten dilated in the cases where the anterior chamber is deep, and in which the tension seems to be due to the increased viscosity of the aqueous; while a contracted pupil is usually present along with a shallow anterior chamber and the hypertension is due to the crowding forward of the iris and evident thlocking of the sinus angle.

Just what influence swelling of the ciliary body has on the production of tension in these cases, is somewhat difficult to determine, tho tenderness in the ciliary region is often most pronounced in the cases which later develop minus tension.

In attempting to isolate the cases in which mydriatic treatment may be logically and safely applied in the presence of + tension, one must take into consideration the following points:

1. The age of the patient (young and middle aged individuals being in the majority, while primary glaucoma is peculiar to advanced age).

2. The history of the case, which in- cludes the absence of prodromal symptoms, which, according to Weeks, are present in about $75 \%$ of the cases of acute glaucoma.

3 . The involvement of one eye only, while primary glaucoma most often affects both eyes.

4. The lack of corneal haziness and anesthesia, but the presence of either cloudy aqueous or other distinct evidence of exudation.

5. In the majority of cases a deep anterior chamber.

Of course, ciliary tenderness and other common evidences of iridocyclitis must be taken into account.

In closing I cannot refrain from observing that while some of these cases may require iridectomy for their relief (e. g. eyes that have annular posterior synechia, or anterior peripheral synechia), I am firmly convinced that some of the cases of so called acute primary glaucoma which are cured by iridectomy belong to this class, which if treated early and vigorously with mydriatics would have made an equally good recovery.

\section{THE ANGLE ALPHA.}

\section{E. LANDOLT, M.D}

PARIS, FRANCE.

This article explains and defends the earlier attempts of its writer to make more definite the understanding of the angle alpha and the factors that influence the apparent diviation of the cornea from its normal position.

In the report of the Committee on "Collective Investigation Concerning the Ocular Muscles" to the Section on Ophthalmology of the American Medical Association, I have just read ( $p$. 277 Presession Volume) the following statement :

"From the carliest days of ophthalmology we have been taught that except in low degree of myopia, the optic axis $\mathrm{AA}^{\prime}$ meets the visual axis $O F$ at an angle $A X O$, which angle has long been known as the angle alpha. ... Unfortunately Landolt did not follow the example of Donders in calling the angle made by the optic with the visual axis the angle alpha. Instead he gave the name to the angle OXI:
The result was that the later writers .. have tried to clear up this confusion by referring to the "angle alpha of l)onders" as one, and the "angle alpha of Landolt as another angle."

The diagram explaining this assertion happens to be taken from my work: "The Refraction and Accommodation of the Eye," Fig. 52, p. 117. I reproduce it here with its original foot note.

It is self evident that the text in my book gives full and clear explanation of these points, lines, angles, etc. Moreover, at the foot of the page I have quoted passages from the works of Dunders, Helmholtz and others, from 